\title{
Diversidad genética en 105 accesiones de cacao (Theobroma cacao L.) colectadas en nicaragua, utilizando 10 marcadores moleculares tipo SSR
}

\section{Genetic diversity in 105 cocoa accessions (Theobroma cacao L.) colected in nicaragua, using 10 molecular markers type SSR}

\author{
Kenia Herrera-García1 ${ }^{1}$, Erwin Aragón-Obando², Víctor Aguilar-Bustamante ${ }^{3}$ \\ ${ }^{1}$ Graduada de la carrera de ingeniería agronómica, ${ }^{2}$ Instituto Nicaragüense de Tecnología Agropecuaria, ${ }^{3}$ Facultad de
} Agronomía, Universidad Nacional Agraria

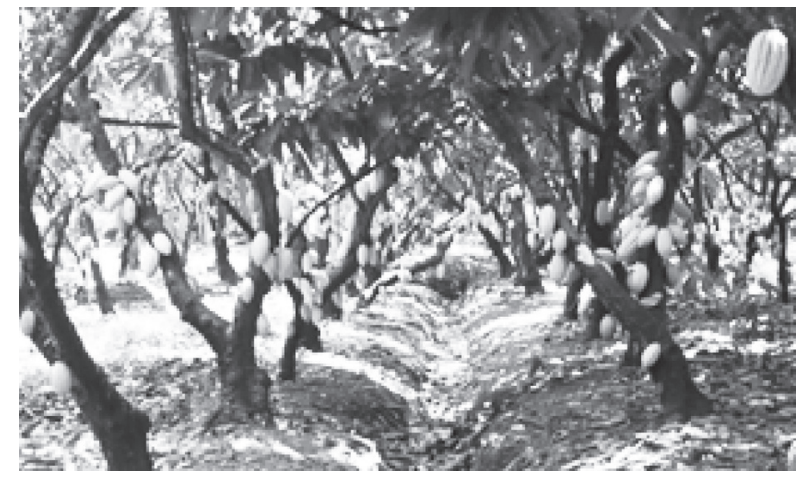

\section{RESUMEN}

Se realizó una caracterización molecular a 105 accesiones de cacao de 79 colectas nacionales, 21 híbridos de referencia y cinco materiales criollos de referencia agrupados en ocho grupos (Rio San Juan, Criollos referencia, híbridos referencia, Carazo, Rivas, Matagalpa, Jinotega y RAAS), utilizando 10 marcadores moleculares tipo SSR con el objetivo de conocer la diversidad genética del cacao colectado en fincas de productores nicaragüenses. Se utilizó el método de extracción de ADN CTAB-Búfer, amplificación de fragmentos de $\mathrm{ADN}$ mediante la técnica de la cadena de reacción de la polimerasa (PCR), visualización de fragmentos de ADN en cámara electroforesis. Se identificaron 183 alelos en las 105 accesiones con una media de 18.3, los rangos de heterosigocidad observada y esperada resultaron mayores para MTcCIR12 con 0.437 y 0.81 respectivamente; La media general del contenido de información polimórfica (CIP) e Índice de Shannon para todos los locus es de 0.87 y 1.37 respectivamente; el análisis molecular de varianza reveló que las diferencias genéticas dentro de los grupos (85\%), mayor que las diferencias entre grupos (15\%). En el análisis de componentes principales (PCA) se encontró cacaos colectados que resultaron genéticamente parecidos a los criollos referencias, es el caso de RSJ0211, RSJ 0311, RSJ 0511, Bomat0510, Ji0210; los materiales Ji-Cuá 0104 y RSJ0411 están estrechamente relacionados. Se demostró que existe una alta diversidad genética y que existen materiales promisorios para posteriores estudios de mejora genética.

Palabras clave: Theobroma cacao, SSR, diversidad genética, heterosigocidad esperada, identidad genética, distancia genética.
A molecular characterization of 105 accessions of cacao (79 nationals, 21 hybrids and 5 creoles) plant was conducted using 10 (SSR) molecular markers. Seventy-nine accessions collected around the country were comprised into 8 groups according to geographic site (Rio San Juan, Creoles reference, hybrids reference, Carazo, Rivas, Matagalpa, Jinotega, RAAS). Twenty-one hybrids and 5 creoles samples were using as reference materials. A total of 183 alleles were identified in 105 accessions with a mean of 18.3. The observed and expected heterosigocity were higher with 0.437 and $0.81 \mathrm{MTc}-$ CIR12, respectively. The means of PIC and Shannon index were 0.87 and 1.37 respectively. The analysis of variance revealed high difference within group (85\%) and low differences among groups $(15 \%)$. The PCA was found collected materials that were genetically similar to creoles references, in the case of RSJ 0211, RSJ 0311, RSJ 0511, Bomat0510, Ji0210, Ji-Cua materials RSJ0411 0104 and are closely related. It was shown that a high genetic diversity and that are promising materials for future breeding studies.

Keywords: Theobroma cacao, Simple Sequence Repeat - SSR, genetic diversity, expected heterosigocity, genetic identity, genetic distance

Recibido: 26 de noviembre 2014

Aceptado: 8 de mayo 2015 
$T$ heobroma cacao L. $(2 \mathrm{n}=20)$, pertenece a la familia de las Esterculeáceas. Es una planta nativa del trópico húmedo. En épocas prehispánicas se cultivaba cacao prácticamente en cualquier sitio que presentara condiciones mínimas para su desarrollo, esto se comprobó con el hallazgo de plantas cultivadas en diversos lugares en el centro de la Península de Yucatán (Millón, 1955). Para la cultura Maya y Azteca, el cacao era usado en ceremonias religiosas y casamientos, como una bebida especial, dándole diferentes usos, preparación y consumo al cacao, caso contrario ocurría con las culturas precolombinas de Sudamérica (McNeil, 2006). Por ello se le atribuye a México y Centro América su centro de domesticación.

Se han reconocido tres grupos genéticos de cacao bien diferenciados, Criollos, Forasteros y Trinitarios. El grupo criollo, se caracteriza por su aroma, la mazorca es de color roja o amarilla cuando madura, presenta alrededor de 10 surcos profundos, muy rugosos, cáscara suave y semillas redondas de medianas a grandes, los cotiledones frescos son de color blanco o violeta pálido, este grupo fue encontrado en México y Nicaragua (Días, 2001). El árbol es de porte bajo y menos robustos que los otros genotipos, tiene bajo rendimiento y se caracteriza por su alta susceptibilidad a las principales enfermedades (Soria, 1966). El grupo forastero produce una mazorca amarilla cuando están maduras y con surcos y rugosidades poco conspicuas, lisas y de extremo redondeado o punta muy corta; las semillas son más o menos aplanadas y los cotiledones frescos son de color violeta (Urquhart, 1963). El cacao tipo trinitario es el resultado de la hibridación natural entre los cacaos criollos y forasteros, ocurrida en el norte de Brasil. Comprenden formas híbridas heterogéneas, su calidad y características botánicas son intermedias entre los criollos y forasteros (Arguello et al., 2000). Son árboles de gran vigor, alta producción y resistencia a enfermedades. Las mazorcas y sus semillas presentan una amplia variabilidad, el color de la mazorca varia en cuanto al tono de morado que ellos presentan.

Nicaragua se ubica en el lugar 42 de los países productores de cacao y participa con un $0.03 \%$ en el comercio mundial. Se estima que hay alrededor de 7500 ha sembradas con cacao, distribuidas en siete grandes núcleos productivos que se ubican en los departamentos de la RAAS, RAAN, Matagalpa, Jinotega, Río San Juan, Granada y Rivas (Orozco, 2010). Aunque en volumen de producción no es significativo, en calidad sí, beneficiado por las condiciones agrogeográficas de nuestro país y la genética misma de los materiales; es por ello la demanda de nuestro cacao en el mercado internacional.

En el presente estudio, se describe la caracterización molecular de 79 accesiones de cacao colectadas a nivel nacional, agregando 21 híbridos y 5 criollos de referencia con el objetivo de identificar las diferencias y relaciones genéticas de la colección del germoplasma nicaragüense, a través del uso de marcadores moleculares tipo microsatélites.

\section{MATERIALES Y MÉTODOS}

Material colectado. Se colectaron 79 accesiones a nivel nacional en las principales zonas donde se encuentran plantaciones de cacao. Se seleccionó material tipo élite (sanos y altamente productivos, alta adaptabilidad a la zona, árboles robustos) y accesiones con características propias de los materiales criollos (árbol pequeño y débil, hojas pequeñas y verde claro, mazorca en forma angoleta o cundeamor, cáscara fina y suave, superficie de la mazorca rugosa, almendras blancas y redondeadas, sabor fino de las almendras y con poca adaptabilidad a la zona). Se consideraron 21 híbridos $\mathrm{y}$ cinco materiales criollos utilizados como referencia y agrupadas en ocho grupos, de acuerdo a su departamento de origen (cuadro 1).

Cuadro 1. Descripción de los tratamientos empleados en el ensayo

\begin{tabular}{cccc}
\hline Entrada & Nombre de accesión & Departamento & Tipo genético \\
\hline 1 & CRZ 0111 & Carazo & Élite \\
2 & CRZ 0211 & Carazo & Elite \\
3 & CRZ 0311 & Carazo & Elite \\
4 & CRZ 0411 & Carazo & Elite \\
5 & CRZ 0511 & Carazo & Elite \\
6 & CRZ 0611 & Carazo & Criollo \\
7 & SJC 0108 & Criollos Referencia & Criollo \\
8 & MENIER 0108 & Criollos Referencia & Criollo \\
9 & RAAS 0403 & Criollos Referencia & Criollo \\
10 & Criollo 13 & Criollos Referencia & Criollo \\
11 & Yucatán CR & Criollos Referencia & Híbrido \\
12 & SHN 8602 & Híbridos Referencia & Híbrido \\
13 & IMC67xUF613 & Híbridos Referencia & Híbrido \\
\hline
\end{tabular}


Cuadro 1. Continuación...

\begin{tabular}{|c|c|c|c|}
\hline 15 & ICS8xP12 & Híbridos Referencia & Híbrido \\
\hline 16 & UF668xIMC67 & Híbridos Referencia & Híbrido \\
\hline 17 & EET162xSCV6 & Híbridos Referencia & Híbrido \\
\hline 18 & SCV6xUF613 & Híbridos Referencia & Híbrido \\
\hline 19 & UF650xIMC67 & Híbridos Referencia & Híbrido \\
\hline 20 & EET400xUF613 & Híbridos Referencia & Híbrido \\
\hline 21 & UF654xP2 & Híbridos Referencia & Híbrido \\
\hline 22 & UF221xIMC67 & Híbridos Referencia & Híbrido \\
\hline 23 & UF677xIMC67 & Híbridos Referencia & Híbrido \\
\hline 24 & GS36xUF296 & Híbridos Referencia & Híbrido \\
\hline 25 & UF296xIMC67 & Híbridos Referencia & Híbrido \\
\hline 26 & EET96xSCV6 & Híbridos Referencia & Híbrido \\
\hline 27 & UF67xP12 & Híbridos Referencia & Híbrido \\
\hline 28 & EET95xSCV6 & Híbridos Referencia & Hibrido \\
\hline 29 & EET399 & Híbridos Referencia & Híbrido \\
\hline 30 & UF613 & Híbridos Referencia & Híbrido \\
\hline 31 & POUND7 & Híbridos Referencia & Híbrido \\
\hline 32 & SPA9 & Híbridos Referencia & Híbrido \\
\hline 33 & Ji 0110 & Jinotega & Criollo \\
\hline 34 & Ji 0210 & Jinotega & Criollo \\
\hline 35 & Cuá 0110 & Jinotega & Criollo \\
\hline 36 & Ji-Cuá 0104 & Jinotega & Criollo \\
\hline 37 & Cuá 0402 & Jinotega & Criollo \\
\hline 38 & wla1001 & Jinotega & Elite \\
\hline 39 & wla1003 & Jinotega & Elite \\
\hline 40 & wla1008 & Jinotega & Elite \\
\hline 41 & wla1019 & Jinotega & Élite \\
\hline 42 & Bomat 0510 & Matagalpa & Criollo \\
\hline 43 & Bomat 0610 & Matagalpa & Criollo \\
\hline 44 & Bomat 0710 & Matagalpa & Criollo \\
\hline 45 & Bomat 0810 & Matagalpa & Criollo \\
\hline 46 & Bomat 0910 & Matagalpa & Criollo \\
\hline 47 & Bomat 1010 & Matagalpa & Criollo \\
\hline 48 & Bomat 1011 & Matagalpa & Criollo \\
\hline 49 & Wamat 0110 & Matagalpa & Criollo \\
\hline 50 & Wamat 0310 & Matagalpa & Criollo \\
\hline 51 & Wamat 0410 & Matagalpa & Criollo \\
\hline 52 & Wamat 01210 & Matagalpa & Criollo \\
\hline 53 & NG 0211 & RAAS & Élite \\
\hline 54 & NG0111 & RAAS & Elite \\
\hline 55 & NG0311 & RAAS & Elite \\
\hline 56 & NG0411 & RAAS & Elite \\
\hline 57 & NG0511 & RAAS & Elite \\
\hline 58 & Corozo & RAAS & Criollo \\
\hline 59 & RIV0111 & Rivas & Elite \\
\hline 60 & RIV0211 & Rivas & Elite \\
\hline 61 & RIV0311 & Rivas & Elite \\
\hline 62 & RIV0411 & Rivas & Elite \\
\hline 63 & RSJ 0211 & RSJ & Elite \\
\hline 64 & RSJ 0311 & RSJ & Elite \\
\hline
\end{tabular}


Cuadro 1. Continuación...

\begin{tabular}{|c|c|c|c|}
\hline 65 & RSJ 0411 & RSJ & Elite \\
\hline 66 & RSJ 0511 & Rio San Juan & Élite \\
\hline 67 & RSJ 0611 & Rio San Juan & Élite \\
\hline 68 & RSJ 0711 & Rio San Juan & Élite \\
\hline 69 & RSJ 0811 & Rio San Juan & Élite \\
\hline 70 & RSJ 0911 & Rio San Juan & Élite \\
\hline 71 & RSJ 1011 & Rio San Juan & Élite \\
\hline 72 & RSJ 1111 & Rio San Juan & Élite \\
\hline 73 & RSJ 1211 & Rio San Juan & Élite \\
\hline 74 & RSJ 1311 & Rio San Juan & Élite \\
\hline 75 & RSJ 1411 & Rio San Juan & Élite \\
\hline 76 & RSJ 1511 & Rio San Juan & Élite \\
\hline 77 & RSJ 1611 & Rio San Juan & Élite \\
\hline 78 & RSJ 1711 & Rio San Juan & Élite \\
\hline 79 & RSJ 1811 & Rio San Juan & Élite \\
\hline 80 & RSJ 1911 & Rio San Juan & Élite \\
\hline 81 & RSJ 2011 & Rio San Juan & Élite \\
\hline 82 & RSJ 2111 & Rio San Juan & Élite \\
\hline 83 & RSJ 2311 & Rio San Juan & Élite \\
\hline 84 & RSJ 2411 & Rio San Juan & Élite \\
\hline 85 & RSJ 2511 & Rio San Juan & Élite \\
\hline 86 & RSJ 2611 & Rio San Juan & Élite \\
\hline 87 & RSJ 2711 & Rio San Juan & Élite \\
\hline 88 & M 0210 & Melchorita & Élite \\
\hline 89 & M 0220 & Melchorita & Élite \\
\hline 90 & La F 01 & La Fortuna & Élite \\
\hline 91 & La F 02 & La Fortuna & Élite \\
\hline 92 & La F 03 & La Fortuna & Élite \\
\hline 93 & $\mathrm{La} \mathrm{F} 04$ & La Fortuna & Élite \\
\hline 94 & La F 05 & La Fortuna & Élite \\
\hline 95 & Mar01 & Las Maravillas & Élite \\
\hline 96 & Mar 0102 & Las Maravillas & Élite \\
\hline 97 & Mar 0104 & Las Maravillas & Élite \\
\hline 98 & Mar 0105 & Las Maravillas & Élite \\
\hline 99 & Mar 0106 & Las Maravillas & Élite \\
\hline 100 & Mar 0107 & Las Maravillas & Élite \\
\hline 101 & Mar 0108 & Las Maravillas & Élite \\
\hline 102 & Mar 0109 & Las Maravillas & Élite \\
\hline 103 & MER 0110 & Las Maravillas & Élite \\
\hline 104 & Mar 0402 & Las Maravillas & Élite \\
\hline 105 & Pentagona & RSJ & Criollo \\
\hline
\end{tabular}

Extracción de ADN. Para la extracción de ADN se utilizó el método de Bromuro de Cetil-Trimetil Amonio (Doyle y Doyle, 1990). Se tomó aproximadamente un $\mathrm{cm} 2$ de hoja de cada material (descritos en el cuadro 1). El ADN se suspendió en $100 \mu l$ de búfer TE y se mantuvo a una temperatura de $-20^{\circ} \mathrm{C}$ hasta su uso.

Amplificación de ADN. Se utilizaron 10 cebadores desarrollados por Lanaud et al., (1999) y seleccionados por Saunders et al., (2004) como cebadores estándar para la caracterización molecular de bancos de germoplasma de cacao (cuadro 2).

Para la reacción de PCR se utilizó un volumen total de $25 \mu$ l; la mezcla contenía 12.5 de Master mix (Taq polimerasa $0.8 \mu \mathrm{l}$, dinucleotidos $0.5 \mu \mathrm{l}$ y determinadas cantidades de magnesio en forma de $\mathrm{MgCl} 2, \mathrm{KCl} 1.5 \mu \mathrm{l}$ ), 2 $\mu 1$ de cebador izquierdo y $2 \mu 1$ cebador derecho, $3 \mu 1$ de ADN Genómico y 5.5 de agua ultra estéril calidad PCR.

La amplificación del ADN se realizó en un termociclador MyGene termal cycler modelo MG 96G con el siguiente programa: la desnaturalización inicial durante cuatro minutos a $94^{\circ} \mathrm{C}$, seguidos de 35 ciclos de desnaturalización por 40 segundos a $94^{\circ} \mathrm{C}$, alineamiento durante 40 segundos a $46^{\circ} \mathrm{C}$ o $51^{\circ} \mathrm{C}$ dependiendo del cebador, extensión a $72^{\circ} \mathrm{C}$ durante un minuto y extensión final a $72^{\circ} \mathrm{C}$ por ocho minutos.

Electroforesis. Se mezcló $5 \mu 1$ de ADN amplificado con 1 $\mu 1$ de búfer de carga $6 \mathrm{x}$, para inyectarlos en gel de agarosa simple al $4.5 \%$, teñida con bromuro de etidio en una cámara de electroforesis que contenía búfer tipo TBE (tris, ácido bórico y EDTA), a la par de las muestras de ADN amplificado se utilizó un marcador de peso molecular de 1000 pares de 
bases, utilizado para determinar el tamaño de las bandas o alelos de cada accesión.

Las muestras se llevaron a la cámara de electroforesis durante 60 minutos a 400 Watts y 120 Voltios. La visualización de las bandas se realizó en una cámara de luz ultravioleta (transluminator High performance UV), posteriormente se procedió a tomar la fotografía de las bandas de ADN amplificadas.

\section{RESULTADOS Y DISCUSIÓN}

Diversidad genética de cacao

Número de alelos por locus. Se identificaron 183 alelos con un promedio de 18.3 alelos por locus en las 105 accesiones evaluadas. MTcCIR12 detectó 35 alelos con una media de 9.25, siendo este el locus más polimórfico y a su vez más informativo, comportándose mejor que el resto de los marcadores, al obtener ADN amplificado. Sereno et al.,

\begin{tabular}{|c|c|c|c|c|c|c|}
\hline Cebador & $\begin{array}{c}\text { Cromosoma } \\
\mathrm{N}^{\circ}\end{array}$ & $\begin{array}{l}\text { 5' Cebador } \\
\text { izquierdo }\end{array}$ & 3' Cebador Derecho & $\begin{array}{l}\mathrm{TA}^{*} \\
\left(\mathrm{C}^{\mathrm{o}}\right)\end{array}$ & $\begin{array}{c}\text { Tamaño } \\
\text { (bp) }\end{array}$ & Motif \\
\hline MTcCIR1 & 8 & $\begin{array}{l}\text { ATGCGAATGAC } \\
\text { AACTGGT }\end{array}$ & $\begin{array}{l}\text { GCTTTCAGTCCT } \\
\text { TTGCTT }\end{array}$ & 51 & $150-167$ & $(\mathrm{GA})_{11}$ \\
\hline MTcCIR6 & 6 & $\begin{array}{l}\text { GATAGCTAAGG } \\
\text { GGATTGAGGA }\end{array}$ & $\begin{array}{l}\text { GGTAATTCAATC } \\
\text { ATTTGAGGATA }\end{array}$ & 46 & $333-357$ & $(\mathrm{GA})_{12}$ \\
\hline MTcCIR7 & 7 & $\begin{array}{l}\text { ATTCTCGCAAAA } \\
\text { ACTTAG }\end{array}$ & $\begin{array}{l}\text { GATGGAAGGAG } \\
\text { TGTAAATAG }\end{array}$ & 51 & $276-301$ & $(\mathrm{TC}){ }_{12} \mathrm{~N}_{146}(\mathrm{CT})_{10}$ \\
\hline MTcCIR8 & 9 & $\begin{array}{l}\text { CTAGTTTCCCAT } \\
\text { TTACCA }\end{array}$ & $\begin{array}{l}\text { TCCTCAGCATTT } \\
\text { TCTTTC }\end{array}$ & 46 & $290-307$ & $(\mathrm{TC})_{5} \mathrm{TT}(\mathrm{TC})_{17} \mathrm{TTT}(\mathrm{CT})_{4}$ \\
\hline MTcCIR11 & 2 & $\begin{array}{l}\text { TTTGGTGATTAT } \\
\text { TAGCAG }\end{array}$ & $\begin{array}{l}\text { GATTCGATTTGA } \\
\text { TGTGAG }\end{array}$ & 46 & $286-321$ & $(\mathrm{TC})_{13}$ \\
\hline MTcCIR12 & 4 & $\begin{array}{l}\text { TCTGACCCCAAA } \\
\text { CCTGTA }\end{array}$ & $\begin{array}{l}\text { ATTCCAGTTAAA } \\
\text { GCACAT }\end{array}$ & 46 & $165-256$ & $(\mathrm{CATA})_{4} \mathrm{~N}_{18}(\mathrm{TG})_{6}$ \\
\hline MTcCIR15 & 1 & $\begin{array}{l}\text { CAGCCGCCTCTT } \\
\text { GTTAG }\end{array}$ & $\begin{array}{l}\text { TATTTGGGATTC } \\
\text { TTGATG }\end{array}$ & 46 & $234-263$ & $(\mathrm{TC})_{19}$ \\
\hline MTcCIR18 & 4 & $\begin{array}{l}\text { GATAGCTAAGG } \\
\text { GGATTGAGGA }\end{array}$ & $\begin{array}{l}\text { GGTAATTCAATC } \\
\text { ATTTGAGGATA }\end{array}$ & 51 & $333-357$ & $(\mathrm{GA})_{12}$ \\
\hline MTcCIR22 & 1 & $\begin{array}{l}\text { ATTCTCGCAAAA } \\
\text { ACTTAG }\end{array}$ & $\begin{array}{l}\text { GATGGAAGGAG } \\
\text { TGTAAATAG }\end{array}$ & 46 & $276-301$ & $(\mathrm{TC})_{12} \mathrm{~N}_{146}(\mathrm{CT})_{10}$ \\
\hline MTcCIR2 & 9 & $\begin{array}{l}\text { TTTGGGGTGATT } \\
\text { TCTTCTGA }\end{array}$ & $\begin{array}{l}\text { TCTGTCTCGTCT } \\
\text { TTTGGTGA }\end{array}$ & 46 & $186-207$ & $(\mathrm{AG})_{13}$ \\
\hline
\end{tabular}

Parámetros evaluados en este estudio

Análisis de diversidad genética. Para conocer la diversidad genética de las accesiones en estudio, se utilizó el software GenAlex v 6.2 como herramienta de apoyo para análisis de datos. Los análisis realizados fueron: número de alelos por locus y grupo, Heterosigocidad observada y esperada por grupo y locus, contenido de información polimórfica por locus.

Análisis de estructura genética. Para los parámetros de diferenciación genética $\mathrm{y}$ análisis de componentes principales, se realizó con el programa GenAlex v 6.2.

Diferenciación genética. La diferenciación genética se cuantificó mediante un análisis molecular de varianza (AMOVA), descritas por Weir y Cockerha (1984), Excoffier et al., (1992) y Weir (1996).

Análisis de componentes principales. Se obtuvo una figura de dos dimensiones donde se compararon individuos entre sí y se agruparon de acuerdo a su relación genética.
(2006) encontraron un promedio de ocho alelos para este mismo cebador y resultó ser el marcador más informativo (cuadro 3).

Los cebadores MTcCIR1 y MTcCIR12 proporcionaron mayor información, en cambio los microsatélites que revelaron menor número de alelos fueron el MTcCIR22 y MTcCIR15 con 14 alelos cada uno (cuadro 3); este a su vez fue el cebador que se comportó recesivo al momento de visualizar el producto PCR y se tuvo que realizar repeticiones de PCR para obtener un número significativo de muestras ampliadas. Efombagn et al., (2006) reportaron que MTcCIR15 identificó 22 alelos, siendo este uno de los marcadores más informativos en su estudio, no así, a los resultados registrados en este estudio.

Heterosigocidad observada y esperada por locus. A mayor heterosigocidad observada en comparación con la heterosigocidad esperada, menor diversidad genética. En este estudio MTcCIR12 y MTcCIR1 presentó el valor promedio más alto (0.43) en cuanto a la heterosigocidad observada, sin embargo, este valor está por debajo del promedio de heterosigocidad esperada para este mismo marcador $(0.81 \mathrm{y}$ 
0.69 respectivamente), lo que indica que para este y el resto de los marcadores utilizados, presenta una alta diversidad genética para todos los locus estudiados (cuadro 3). Romero et al., (2010) reportaron datos de Hobs de 0.39 y de Hesp de 0.80 para MTcCIR12 y fue el cebador que registró los valores más bajos de diversidad genética en híbridos de cacao en Ecuador.

El marcador que detectó menor heterosigocidad esperada en este estudio fue MTcCIR15 (0.58). Aragón (2009) encontró para este mismo marcador, una heterosigocidad esperada de 0.82 y le resultó el marcador más informativo; sin embargo, en este estudio este marcador se comportó recesivo al amplificar ADN y se omitió la lectura de algunas muestras que podrían haber sido heterocigotos (cuadro 3).
Esto corrobora lo que Saunders et al., (2004) menciona acerca de la efectividad de los marcadores para cacao, utilizando en este estudio ocho de los que consideró mejores para análisis de diversidad genética y dos de los que le resultaron ser los más difíciles de amplificar (MTccCIR6, MTcCIR12), pero que en este estudio son los que mejor se comportaron y que brindaron mayor información.

Se demuestra que MTcCIR12 es el más informativo y polimórfico, registra los valores más altos en cuanto a CIP y número de alelos por locus, es efectivo en análisis de diversidad genética sin importar la calidad de ADN que se utilice y el número de muestras amplificadas. MTcCIR12 después de MTcCIR1 fue el cebador que amplificó el mayor número de muestras sin repeticiones. Para el total de muestras estudiadas en comparación al número de marcadores utilizados en este estudio, se encontró un alto nivel de polimorfismo; Cryer 0 ., (2006) consideran a estos marcadores importantes y altamente representativos para la especie, añadiéndole lo mencionado por Saunders et al., (2004) al considerar que estos marcadores son más que suficientes para caracterizar bancos de germoplasma de cacao alrededor del mundo.

Número de alelos por grupo. El grupo que presentó el mayor número de alelos fue Rio San Juan con un promedio de

13.2, dado que en esta zona, predominan plantaciones jóvenes observada, CIP: Contenido de información polimórfica.

Los valores promedios de heterosigocidad observada para todos los locus es de 0.209 y para la heterocigosidad esperada es de 0.663 , y se debe a que ningún microsatélite utilizado para este estudio reveló una heterosigocidad observada menor que el de heterosigocidad esperada; Zhang et al., (2009) registraron 0.51 de heterosigocidad esperada y 0.46 heterosigocidad observada en clones mejorados, sin embargo, en este estudio se utilizaron materiales pertenecientes a los grupos genéticos trinitarios y forasteros en su mayoría y en menor proporción criollos y mejorados; lo que explica porque encontramos mayor diversidad genética.

Contenido de información polimórfica. El contenido de información polimórfica, mostró un valor mayor de 0.5 , demostrando que los microsatélites utilizados son altamente informativos (cuadro 3). El MTcCIR12 presentó el valor más alto de 0.96 y la mayoría de los marcadores utilizados en este estudio presentan valores altos, MTcCIR6 con un valor de 0.78 siendo este el valor más bajo. Romero et al., (2010) reportan un CIP bajo para este mismo cebador (0.71), en cambio el valor más alto se lo proporcionó MTcCIR15 (CIP $=1.00$ ). provenientes de programas de mejoramiento genético. El grupo Rivas es el que obtuvo el menor promedio de alelos con 2.6 (cuadro 4).

Heterosigocidad observada y esperada por grupo: El grupo Rio San Juan presenta el valor promedio más alto en cuanto a la heterosigocidad observada, siendo de 0.313 , sin embargo, no mayor que el de la heterosigocidad esperada que es de 0.863 siendo el rango mayor. El grupo Matagalpa presenta el menor valor promedio de heterosigocidad observada (0.08) no mayor que el de la esperada que es de 0.69 , pero para este mismo parámetro el valor promedio más bajo de heterosigocidad esperada lo presentó el grupo Rivas con 0.50 , determinando una diversidad genética media de acuerdo a Aragón (2009), quien indican que si la heterosigocidad esperada es menor a 0.50 , la diversidad genética es baja.

En el grupo Rivas pueden encontrarse materiales homocigotos, características propias del grupo genético criollo y es el grupo donde se encontró menor número de alelos. Por ello, el valor promedio del grupo criollo referencia presenta una media de 0.58 , parecido al del grupo Rivas que es de 0.50 (cuadro 4 ). 
Cuadro 4. Parámetros para diversidad genética basada en los grupos estudiados

\begin{tabular}{lcccc}
\hline Locus & $\mathrm{N}$ & $\mathrm{NA}$ & $\mathrm{H}_{\text {obs }}$ & $\mathrm{H}_{\text {esp }}$ \\
\hline Rio San Juan & 43 & 13.20 & 0.31 & 0.86 \\
Híbridos referencias & 21 & 7.60 & 0.20 & 0.72 \\
Matagalpa & 11 & 5.20 & 0.08 & 0.69 \\
Jinotega & 9 & 6.20 & 0.22 & 0.78 \\
RAAS & 6 & 3.70 & 0.19 & 0.54 \\
Carazo & 6 & 4.00 & 0.30 & 0.60 \\
Criollo Referencia & 5 & 3.20 & 0.11 & 0.58 \\
Rivas & 4 & 2.60 & 0.23 & 0.50 \\
\hline MEDIA & 105 & 5.71 & 0.20 & 0.66 \\
\hline
\end{tabular}

N: Número de individuos, NA: Alelos por grupo, Hesp: Heterosigocidad esperada, Hobs: Heterosigocidad observada

\section{Estructura genética}

Análisis molecular de varianza (AMOVA). Este parámetro determinó la estructura genética de los materiales en estudio, reveló que las diferencias genéticas mayores están dentro de los grupos con un $85 \%$ y que las diferencias entre grupo fue de $15 \%$ (cuadro 5), demostrando que aunque se hayan agrupado las accesiones según su sitio de colecta, las diferencias genéticas entre individuos son grandes y las diferencias entre grupos son menores porque en el caso de cacao solo existen tres grupos genéticos que están distribuidos en todo el país, y por tanto baja diferenciación. Zhang et al., (2007) realizaron un estudio para determinar la variación en la estructura genética del cacao ecuatoriano, reportando en el AMOVA una variación dentro de los grupos de $76 \%$.

Según Silvertown y Charlesworth (2001), la naturaleza endémica de un cultivo provoca baja variación entre grupo. Por tanto, para cacao este resultado demuestra la existencia de poblaciones aisladas y que la baja variación entre grupo se debe a la naturaleza misma de este cultivo, que es propio de Centro y Sur América. dos se ubicaron dos materiales genéticamente relacionados Jinotega 0110 (33 en la figura 1) y RSJ 0411 (65 en la figura $1)$.

En el eje tres se encuentran los materiales mejorados en su mayoría. El material Rivas0111 (59 en la figura 1), se relaciona con el híbrido UF6667xIMC67 (23 en la figura 1). Thienhaus (2008) concluye que a partir de la década de los 70 los híbridos de cacao fueron el principal material de siembra en Nicaragua, estos fueron introducidos desde Centro de Agricultura Tropical de Investigación y Enseñanza (CATIE) y Colombia. La estrecha relación del material de Rivas con el híbrido se debe a que este último es el que mejor se adapta a la zona, ya sea el padre o el cruce.

En este mismo eje se encuentran los materiales mejorados en su mayoría; la relación más estrecha que se observa en la figura 1, es la de los híbridos EET162 x SCV6, EET400 x UF613 y UF654 x P2 (17, 20 y 21) con el material Rivas0411 (62). Los híbridos hoy en producción provienen principalmente de las fases de establecimiento después de 1990 con mejores selecciones que en los años70's y 80's.
Cuadro 5. Análisis molecular de varianza para cacao basado en las colectas realizadas

\begin{tabular}{lccccc}
\hline Fuente & GL & SS & MS & VE & \% variación \\
\hline Entre grupo & 7 & 293.5 & 41.9 & 2.4 & $15 \%$ \\
Dentro de grupo & 97 & 1371.2 & 14.1 & 14.1 & $85 \%$ \\
Total & 104 & 1664.7 & & 16.6 & $100 \%$ \\
\hline
\end{tabular}

GL: Grados de libertad, SS: Suma de cuadrados, MS: Media de la suma; VE: Variación estándar.

Análisis de componentes principales. En la figura 1, en el eje uno se encuentran los materiales criollos referencia en su mayoría, RAAS0403 (9 en la figura 1) se relaciona con RSJ0311 (64 en la figura 1), Bomat0511 (Matagalpa 42) y con Rio San Juan 0511 (66 en la figura 1); estos materiales son de diferentes zonas geográficas del país y su relación genética puede estar relacionada al intercambio de materiales entre los productores. El CR10 (criollo 13) y el CR11 (Yucatán) se relaciona con RSJ0211 (63 en la figura 1); el CR10 se relaciona con Ji0210 (Jinotega, 34 en la figura 1). En el eje
En el eje cuatro se ubican la mayor parte de los materiales Rio San Juan, los materiales criollos referencia. SJC0108 (7) relacionado con RSJ1311, RSJ2111 (74 y 82) y el criollo MENIER0108 (8) está relacionado con el RSJ2011 (81). Según Menocal (2006), las primeras plantaciones grandes de cacao se reportaron en el valle de MENIER, por tanto, la relación genética de estos materiales puede acreditársele a las migraciones humanas de la época.

En este mismo eje se encontró un híbrido referencia que es SHN8602, relacionado con el material RSJ2311 (83), este a su vez se relaciona con el criollo 7; se le acredita a que este híbrido, a diferencia del resto que se utilizaron para este estudio, es producto de un cruce de material forastero con un material criollo. 


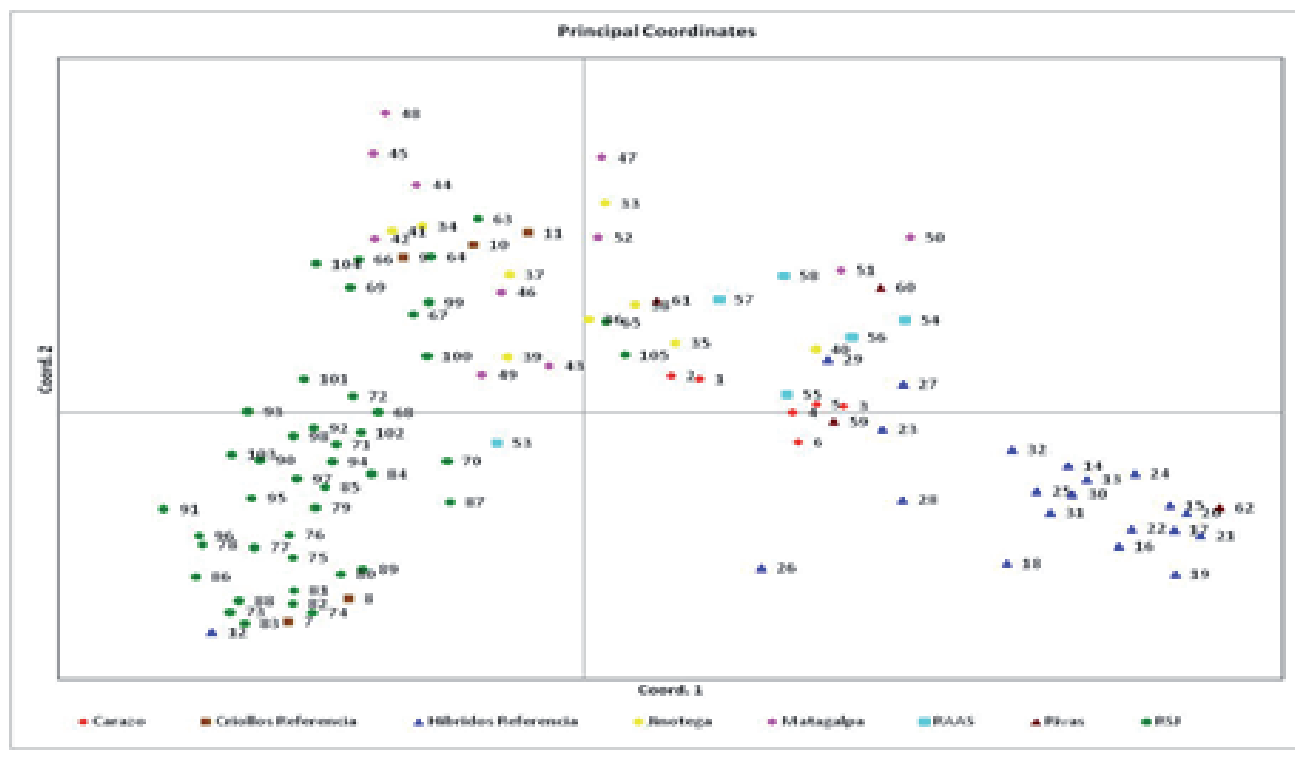

Figura 1. Distribución de las accesiones de cacao según el análisis de componentes principales.
Las relaciones genéticas reveladas en estos resultados muestran que las variaciones y diferencias genéticas de mayor importancia se encuentran dentro de los grupos estudiados y que las diferencias como grupos son menores; por tanto los individuos son diferentes entre sí independiente del lugar donde se encuentre geográficamente.

En el análisis de componentes principales (PCA), las accesiones Bomat0710, Bomat0810 y Bomat1110 de Matagalpa, formaron un grupo diferente al resto de las accesiones evaluadas, se pueden describir como ligadas genéticamente, pero diferentes al resto de accesiones evaluadas.

Se encontraron materiales criollos que fueron estrechamente relacionados con los utilizados como criollos referencias; pero la mayoría de los materiales en este estudio resultaron ser de los otros grupos genéticos.

Se encontraron materiales híbridos relacionados con materiales colectados, indicando alta adaptación de estos híbridos en determinadas zonas del país.

los microsatélites que se utilizaron en este estudio resultaron ser altamente informativos y polimórficos porque no se registraron valores menores a 0.50 .

\section{REFERENCIAS BIBLIOGRÁFICAS}

Aragón, EM. 2009. Genetic characterization of Theobroma cacao L. in Nicaragua. Thesis MSc. University of Helsinki, Departament of Applied Biology. Helsinki. FL. 49 p.

Arguello, O; Mejía, L; Palencia, C. 2000. Origen y descripción botánica. In Tecnología para el mejoramiento de sistemas de producción de cacao, Corpoica, Bucaramanga, CO. p 10-12.

Cryer, NC; Fenn, M.G.E; Turnbull, CJ; Wilkinson, MJ. 2006. Allelic size standards and reference genotypes to unify international cocoa (Theobroma cacao L.) microsatellite data. Genetic Resources and Crop Evolution 53:1643-1652.

Días L, AS. 2001. Genetic improvement of cacao (melhoramento genético do cacaueiro) (ed. Dias LAS), FUNAPE-UFG, Brazil. Translated into English by Abreu-Richart CE (http://ecoport.org).

Doyle, JF; Doyle, JL. 1990. Isolation of plant DNA from fresh tissue. Focus 12:13-15.

Efombagn, M,I,B; Sounigo, O; Manzanares, M; Cilas, C; Eskes, M.A.B; Allen, K. 2006. Genetic diversity in cocoa germoplasm southern Cameroon revealed by simple sequences repeat (SSRS) markers. African Journal of Biotechnology. 5(16):1441-1449.

Excoffier, L; Smouse, P; Quattro, J. 1992. Analysis of molecular variance inferred from metric distances among DNA haplotypes: Application to human mitochondrial DNA restriction data. Genetics 131(2):479-491.

Lanaud, C; Risterucci, AM; Pieretti, I; Falque, M; Bouet, A; Lagoda P, JL. 1999. Isolation and characterization of microsatellites in Theobroma cacao L. Molecular Ecology. 8:2141-2152.

McNeil, C. 2006. Chocolate in Mesoamerica. A cultural history of cacao. University Press of Florida. 542 p.

Menocal, O. 2006. El cacao: Riqueza potencial de la tierra nica a la espera de ser explotada comercialmente en los mercados internacionales. Instituto Nicaragüense de Tecnología Agropecuaria (INTA). Dirección de Investigación y Desarrollo. DSAINTA. 7 p.

Millon, R. 1955. When the money grew on the trees. A study of cacao in ancient Mesoamerica, thesis doctoral, Columbia University, University of Microfilms, Ann Arbor. 12 p.

Orozco, L. 2010. Mapa cacaotero de Nicaragua: zonas productivas, organizaciones de productores, agencias de apoyo y exportadores. PCC. 26 p. 


\section{REEURSOSGENADTLOS}

Romero, CA; Bonilla, JA; Santos, EG; Peralta, EL. 2010. Identificación varietal de 41 plantas seleccionadas de cacao (Theobroma caco L.) provenientes de cuatro cutivares distintos de la región amazonoca ecuatoriana, mediante el uso de microsatélites. Revista Tecnologica ESPOL 23(1):121-128.

Saunders, JM; Mischke, S; Leamy, EA; Hemeida, AA. 2004. Selection of international molecular standards for DNA fingerprinting of Theobroma cacao. Theoretical and Applied Genetics 110:41-47.

Sereno, ML; Alburquerquer P, SB; Vencovsky, R; Figueira, A. 2006. Genetic diversity and natural population structure of cacao (Theobroma cacao L.) from the Brazilian Amazon evaluated by microsatellite markers. Conservation genetics 7:13-24.

Silvertown, J; Charlesworth, D. 2001. Plant population biology. Blackwell publishing. ed. 4. 302 p.

Soria, VJ. 1966. Obtención de clones de cacao por el método de índices de selección. Instituto Interamericano de Cooperación para la Agricultura, Turrialba, CR. 16:119-124.

Thienhaus, S, 2008. Composición genética de las plantaciones híbridas de cacao. El Recreo. Diagnóstico de la producción de cacao en Nicaragua. 25p.

Urquhart, DH. 1963. Cacao. Instituto del libro. Edición revolucionaria. Habana, CU. 322 p.

Weir, BS. 1996. Genetic data analysis II.Sinauer, Sunderland, Massachusetts, USA.

Weir, BS; Cockerham, CC. 1984. Estimating F-statistics for the analysis of population structure. Evolution 38:1358-1370.

Zhang, D; Boccara, M; Motllal, L; Butler, DR; Umahoran P; Mishk, S; Lyndel, M. 2007. Microsatellite variation and population structure in the refractorio cacao of Ecuador. ConsevGenet. 9:327-337.

Zhang, D; Mischke, S; Johnson, ES; Phillips Mora, W; Meinhardt, L. 2009. Molecular characterization of an international cacao collection using microsatellite markers. Tree Genetics \& Genomes 5:1-10. 